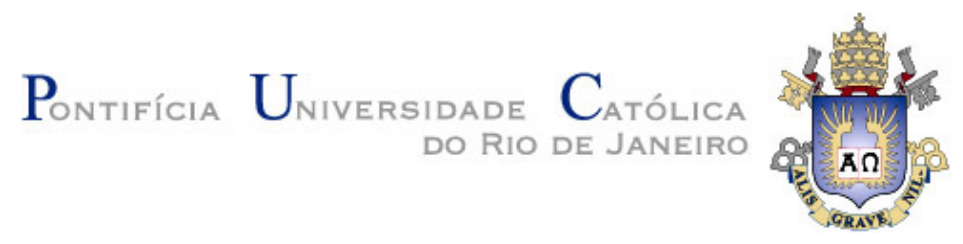

Thiago Valente Aguiar

\title{
Visualização da Fronteira entre Fluidos utilizando o Método SPH e o Algoritmo de Marching Cubes
}

\section{Dissertação de Mestrado}

Dissertação apresentada ao Programa de Pós-graduação em Informática da PUC-Rio como requisito parcial para obtenção do título de Mestre em Informática.

Orientador: Prof. Bruno Feijó

Rio de Janeiro Setembro de 2009 


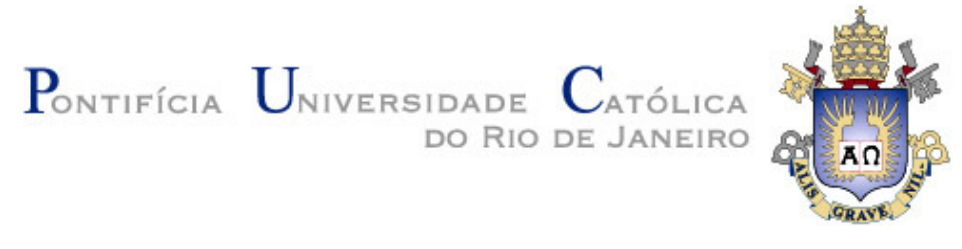

Thiago Valente Aguiar

\section{Visualização da Fronteira entre Fluidos utilizando o Método SPH e o Algoritmo de Marching Cubes}

Dissertação apresentada como requisito parcial para a obtenção do grau de Mestre pelo Programa de Pósgraduação em Informática do Departamento de Informática do Centro Técnico e Científico da PUC-Rio. Aprovada pela Comissão Examinadora abaixo assinada.

Prof. Bruno Feijó Orientador Departamento de Informática - PUC-Rio

Prof. Alberto Barbosa Raposo Departamento de Informática - PUC-Rio

Prof. Marcelo de Andrade Dreux Departamento de Engenharia Mecânica - PUC-Rio

Prof. José Eugenio Leal Coordenador Setorial do Centro

Técnico Científico - PUC-Rio

Rio de Janeiro, 17 de setembro de 2009 
Todos os direitos reservados. É proibida a reprodução total ou parcial do trabalho sem autorização da universidade, do autor e do orientador.

Thiago Valente Aguiar

Graduou-se em Ciência da Computação pela Universidade Federal de Viçosa. Desenvolveu como projeto de mestrado um método sobre a visualização de fronteira entre fluidos simulados por partículas. Trabalhou junto ao Tecgraf - PUC-Rio na área de Computação Gráfica e Automação de Projetos.

Ficha Catalográfica

Aguiar, Thiago Valente.

Visualização de Fronteira entre Fluidos utilizando o Método SPH e o Algoritmo de Marching Cubes / Thiago Valente Aguiar; orientador: Bruno Feijó. - Rio de Janeiro : PUC-Rio,

Departamento de Informática, 2009

v., 58 f: il. ; 29,7 cm

1. Dissertação (mestrado) - Pontifícia Universidade Católica do Rio de Janeiro, Departamento de Informática.

Inclui referências bibliográficas.

1. Informática - Dissertação. 2. Computação Gráfica. 3. Fronteira entre Fluidos. 4. SPH. 5. Marching Cubes. I. Feijó, Bruno. II. Pontifícia Universidade Católica do Rio de Janeiro. Departamento de Informática. III. Título 
Dedico este trabalho à minha mãe, Terezinha, pelo apoio aos estudos em todas as fases da minha vida. 


\section{Agradecimentos}

Ao meu orientador, professor Bruno Feijó, por me ajudar a traçar os caminhos deste trabalho.

Aos membros da banca examinadora, pelo direcionamento e avaliação deste estudo.

Ao CNPq pelo auxílio financeiro que me foi dado.

Ao laboratório Tecgraf pela oportunidade que me foi dada como pesquisador.

À minha família que sempre me apoiou nos estudos, em especial à minha mãe.

Ao meu pai e meu avô pela constante inspiração.

Às minhas irmãs por me darem uma força e um auxlílio num momento tão necessário.

Aos meus amigos pela força e estímulo em finalizar mais esta etapa.

Ao meu amigo e irmão Vinícius Lopes Rodrigues pelo companheirismo em todas as fases de meu estudo. 


\section{Resumo}

Aguiar, Thiago Valente.; Feijó, Bruno.. Visualização da Fronteira entre Fluidos utilizando o Método SPH e o Algoritmo de Marching Cubes. Rio de Janeiro, 2009. 58p. Dissertação de Mestrado - Departamento de Informática, Pontifícia Universidade Católica do Rio de Janeiro.

A modelagem de fluidos é frequentemente utilizada, tanto na engenharia como na área de efeitos especiais para cinema e TV, para simular fenômenos naturais, tais como fogo, explosões, fumaça, água e ar. Basicamente, esta modelagem utiliza métodos baseados em malhas, tais como volumes finitos e elementos finitos, ou métodos independentes de malhas baseados em partículas, tais como MPS e SPH. Uma questão comum nas técnicas de partículas é o problema de fronteiras entre fluidos, já que não há uma solução geral e satisfatória. O objetivo deste trabalho é propor uma nova técnica para a visualização da fronteira entre fluidos utilizando o método SPH (Smoothed-particle Hydrodynamics) e o algoritmo Marching Cubes. Testes são realizados para averiguar os custos computacionais envolvidos na geração e renderização da malha da fronteira.

\section{Palavras-chave}

Simulação de fluidos; SPH; Marching Cubes; Fronteira entre Fluidos. 


\begin{abstract}
Aguiar, Thiago Valente.; Feijó, Bruno.. Visualization of the boundary between Fluids using the SPH method and the Marching Cubes algorithm. Rio de Janeiro, 2009. 58p. MScThesis - Department of Computer Science, Pontifícia Universidade Católica do Rio de Janeiro.
\end{abstract}

In engineering and special effects for film and television, fluid modeling is frequently used to simulate natural phenomena, such as fire, explosions, smoke, water, and air. Basically, this modeling uses mesh-based methods, such as finite volumes and finite elements, or mesh-free methods based on particles, such as MPS and SPH. A common question among particle techniques is the problem of boundary between fluids, since there is no general and satisfactory solution available. The objective of this work is to propose a new technique for the visualization of the boundary between fluids using the SPH method (Smoothed-particle Hydrodynamics) and the Marching Cubes algorithm. Tests are made to investigate the computational costs involved in the generation and rendering of the boundary mesh.

\title{
Keywords
}

Fluid simulation; SPH; Marching Cubes; Boundary between fluids 


\section{Sumário}

1 Introdução 14

2 Trabalhos Relacionados $\quad 17$

$\begin{array}{lll}2.1 \mathrm{SPH} & 17\end{array}$

2.2 Visualização Volumétrica 19

2.2.1 Rendering Direto $\quad 19$

2.3 Extração de Superfícies 20

2.4 Marching Cubes $\quad 22$

$3 \mathrm{SPH} \quad 25$

$\begin{array}{lll}3.1 & \text { Introdução } & 25\end{array}$

3.2 Equações Governantes $\quad 26$

$\begin{array}{lll}3.3 & \text { Função de Suavização } & 27\end{array}$

3.4 Modelagem das Equações $\quad 28$

3.4.1 Força de Pressão $\quad 29$

3.4.2 Força de Viscosidade $\quad 30$

3.4.3 Força da Gravidade $\quad 30$

3.4.4 Força de Tensão Superficial 30

4 Marching Cubes 32

4.1 Introdução 32

4.2 O Algoritmo 32

4.2.1 Marching Squares $\quad 32$

4.2.2 Marching Cubes $\quad 34$

5 Sistema Proposto 37

$\begin{array}{ll}5.1 & 37 \text { Identificação da Interface }\end{array}$

5.2 Geração da Malha de Triângulos 38

5.3 Suavização da Malha 41

6 Resultados 45

6.1 Identificação de Partículas da Interface 46

6.2 Malha sem Suavização $\quad 47$

$\begin{array}{ll}\text { 6.3 Malha com Suavização } & 47\end{array}$

6.4 Custo Computacional 48

7 Conclusão e Trabalhos Futuros 51

8 Bibliografia 53

$\begin{array}{ll}\text { Apêndice } & 57\end{array}$ 


\section{Lista de Figuras}

Figura 1 Separador de Produção. Imagem cedida pela Petrobras 15

Figura 2 - Simulação de SPH realizada no trabalho de Müller (2003) 18

Figura 3 - Esquema do algoritmo de Ray-Casting. Os cubos menores representam as frações das cores e opacidades acumuladas do volume de dados

Figura 4 - Representação do algoritmo Conexão de Contonos. Conexão das iso-linhas entre os planos de corte ou "fatias" 21

Figura 5 - Esquerda: Imagem de uma iso-superfície ideal. Direita: Imagem gerada pelo algoritmo de "Cuberille"

Figura 6 - Torus interligados gerados pelo algoritmo de Chernyaev (à esquerda) e pelo de Lewiner (à direita) - Imagem retirada de Lewiner et al (2003)

Figura 7 - Representação do raio de influência de uma partícula

Figura 8 - Esquerda: Partícula no interior do fluido sofre forças por todos os lados. Direita: Partícula da superfície não recebe forças em todos os lados, gerando a força de tensão superficial

Figura 9 - Representação de uma iso-superfície

Figura 10 - Exemplo da iso-linha gerada com o algoritmo Marching Squares com $\mathrm{N}=0$. Os números em cada vértice representam o seu peso $\mathrm{W}$

Figura 11 - Lookup table do algoritmo Marching Squares. Pontos pretos representam valores positivos e brancos valores negativos

Figura 12 - Ambiguidade apresentada pela lookup table do algoritmo Marching Squares

Figura 13 - Planos de corte de um cubo lógico utilizado no algoritmo 
de Marching Cubes

Figura 14 - Construção da superfície através de vértices do cubo

Figura 15 - Padrões estabelecidos por Lorensen (1987) para formar a lookup table

Figura 16 - Imagens de dois torus geradas a partir do algoritmo de Lewiner (2003). $\mathrm{Na}$ esquerda: a superfície construída. $\mathrm{Na}$ direita: a representação da malha

Figura 17 - Identificação de partículas que fazem parte da interface entre os fluidos

Figura 18 - Influência de partículas no cálculo da equação 3.19. Na esquerda: partículas dentro do fluido influenciam partículas vizinhas de mesmo polo. Na direita: partículas da interface influenciam partículas vizinhas de polos diferenciados

Figura 19 - Representação da separação das partículas e distinção das partículas pertencentes a iso-superfície

Figura 20 - Representação bidimensional dos vértices representantes das partículas da interface no grid

Figura 21 - Representação bidimens ional da influência de vértices representantes em vértices vizinhos

Figura 22 - Detalhe do raio de influência

Figura 23 - Malha com extremidades de aparência cúbica

Figura 24 - Representação dos vértices vizinhos numa malha de triângulos

Figura 25 - Representação da nova posição do vértice e a referência de sua posição antiga com uma iteração do Laplaciano 42

Figura 26 - Malha após o algoritmo de suavização Laplaciano 43

Figura 27 - Apresentação das normais dos vértices da malha de triângulos após a suavização

Figura 28 - Apresentação detalhada das normais dos vértices da malha de triângulos após a suavização

Figura 29 - Simulação de dois fluidos imiscíveis utilizando a biblioteca de Nakamura (2007)

Figura 30 - Vista horizontal das partículas candidatas a fazerem parte 
da interface

Figura 31 - Vista por cima das partículas candidatas a fazerem parte da interface

Figura 32 - Resultado obtido com a biblioteca de Lewiner et al (2003) 47

Figura 33 - Detalhe para um retângulo perdendo área quando se torna chanfrado

Figura 34 - Comparação entre malhas. Esquerda: malha sem suavização. Direita: malha após a suavização

Figura 35 - Esquerda: Simulação do SPH com "metaballs". Direita: Resultado da malha obtida da simulação após a suavização 48

Figura 36 - Gráfico que compara a eficiência da geração da malha na renderização de quadros na simulação

Figura 37 - Gráfico que compara a eficiência da suavização na renderização de quadros na simulação

Figura A1 - Gráficos da função de suavização 3.14 e de suas derivadas de primeira e segunda ordem

Figura A2 - Gráficos da função de suavização 3.16 e de suas derivadas de primeira e segunda ordem

Figura A3 - Gráficos da função de suavização 3.18 e de suas derivadas de primeira e segunda ordem 


\section{Lista de Tabelas}

Tabela 1 - Comparação das taxas de quadros por segundo dos 3 modos 


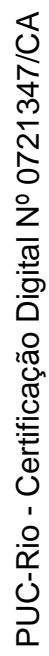

Happiness is not an absence of problems; but the ability to deal with them

Jack Brown 